\title{
Sodium-Glucose Cotransporter 2 (SGLT2) Inhibition in Kidney Transplant Recipients with Diabetes Mellitus
}

\author{
Moritz Mahling ${ }^{a-c}$ Anja Schork ${ }^{a-c}$ Silvio Nadalin ${ }^{d} \quad$ Andreas Fritsche ${ }^{a-c}$ \\ Nils Heyne ${ }^{a-c}$ Martina Guthoff ${ }^{a-c}$ \\ aDepartment of Diabetology, Endocrinology, Nephrology, Section of Nephrology and \\ Hypertension, University of Tübingen, Tübingen, Germany; ${ }^{b}$ Institute for Diabetes Research \\ and Metabolic Diseases of the Helmholtz Center Munich at the University of Tübingen, \\ Tübingen, Germany; ' German Center for Diabetes Research (DZD e.V.), Neuherberg, \\ Germany; ${ }^{d}$ Department of General, Visceral and Transplant Surgery, University of Tübingen, \\ Tübingen, Germany
}

\section{Keywords}

Kidney transplantation · Diabetes mellitus · Sodium-glucose cotransporter 2 inhibition . Empagliflozin · Renal and cardiovascular endpoints

\begin{abstract}
Background: Sodium-glucose cotransporter 2 (SGLT2) inhibition has been shown to reduce cardiovascular mortality and preserve kidney function in patients with type 2 diabetes. Kidney transplant recipients with diabetes demonstrate increased risk and accelerated progression of micro- and macrovascular complications and may specifically benefit from SGLT2 inhibition. However, potential concerns of SGLT2 inhibition include volume depletion and urinary tract infections. Objectives: We report data on the use of SGLT2 inhibitors in a case series of ten patients with diabetes after kidney transplantation in order to analyze efficacy, safety, and the effect on renal function. Methods: Patients with a stable allograft function and no history of recurrent urinary tract infections were eligible. The SGLT2 inhibitor empagliflozin was given as add-on to preexisting antidiabetic treatment with initial dose reduction of the latter. Results: Median estimated glomerular filtration rate at baseline was $57 \mathrm{~mL} / \mathrm{min} / 1.73 \mathrm{~m}^{2}$ and remained stable throughout the follow-up of 12.0 (5.3-12.0) months. Median $\mathrm{HbA}_{1 \mathrm{c}}$ decreased from 7.3 to $7.1 \%$. The rate of urinary tract infections and other side effects was low. Conclusions: SGLT2 inhibition is feasible and well tolerated in selected kidney transplant recipients with diabetes. Whether SGLT2 inhibition is able to reduce cardiovascular mortality and improve allograft survival in these patients has to be addressed in further studies.
\end{abstract}




\section{Kidney \\ Blood Pressure \\ Research}

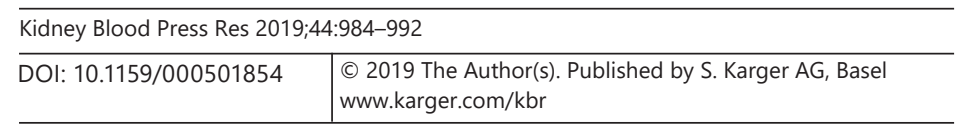

Mahling et al.: SGLT2 Inhibition after Kidney Transplantation

\section{Introduction}

Diabetes mellitus and kidney transplantation are two conditions with a well-established mutual relationship. Diabetes mellitus is the leading cause of end-stage renal disease and thus highly prevalent in kidney transplant recipients [1]. In addition to a preexisting diabetes mellitus, posttransplantation diabetes mellitus yields a significant morbidity and mortality in patients after kidney transplantation [2,3]. Those patients demonstrate an increased risk and accelerated progression of diabetic micro- and macrovascular complications [4]. Furthermore, poor glycemic control is a risk factor for delayed graft function [5] and might thus negatively affect allograft survival [6]. Adequate glycemic control is therefore of major interest when caring for kidney transplant recipients, though complicated by an altered glucose metabolism as a consequence of immunosuppression, the risk of infections, and reduced glomerular filtration rate (GFR) $[7,8]$.

For patients with diabetes mellitus, sodium-glucose cotransporter 2 (SGLT2) inhibition is an emerging therapeutic option. SGLT2 is located in the early proximal tubule of the kidney, reabsorbing glucose from the glomerular filtrate [9]. Selective SGLT2 inhibition results in substantial amounts of urinary excreted glucose, thereby lowering blood glucose levels independent of insulin [9].

Empagliflozin has been shown to reduce both cardiovascular death [10] and progression of diabetic nephropathy [11]. These effects seem to be (at least partly) independent of glucose lowering effects [12]. Possible explanations for the renal protective effects include a reduced glomerular capillary pressure [13] and an amelioration of glomerular hyperfiltration [14]. Recent data suggest that the effect of SGLT2 inhibition is additional to the effects of angiotensin-converting enzyme inhibitors/angiotensin receptor blockers [15]. Further extrarenal effects of SGLT2 inhibition include reductions in body weight and blood pressure, possibly augmenting renoprotective effects [10]. Similar nephroprotective effects have been described for canagliflozin and dapagliflozin, other SGLT2 inhibitors [16, 17], indicating the presence of a class effect. Thus, SGLT2 inhibitors are a promising drug class, yielding a reduction of micro- and macrovascular complications and an enormous potential in the high-risk group of kidney transplant recipients.

On the other hand, SGLT2 inhibition carries the potential risk of side effects including volume depletion and urinary tract infection due to glucosuria [18], both to which kidney transplant recipients are prone. This is further complicated by the complex situation including the necessity of immunosuppression and a single functioning kidney. One simultaneously performed and recently published study showed that SGLT2 inhibition in kidney transplant recipients was feasible [19]. As broader safety data on the use of the SGLT2 inhibitors in kidney transplant recipients are missing to date, SGLT2 inhibitors are currently seldom used in kidney transplant recipients with diabetes, despite the need for inhibition of progression, especially in these patients. At our institution, we started to treat selected kidney transplant recipients with diabetes mellitus with empagliflozin in 2017 and conducted a prospective observational study in kidney transplant recipients on treatment with empagliflozin. We here report data on the use of the SGLT2 inhibitor empagliflozin in a case series of ten patients with diabetes after kidney transplantation in order to assess efficacy, safety, and effect on allograft function.

\section{Materials and Methods}

\section{Study Design and Ethics}

We carried out a prospective observational study in kidney transplant recipients with type 2 diabetes or posttransplantation diabetes mellitus who started treatment with 


\section{Kidney \\ Blood Pressure \\ Research}

Table 1. Baseline characteristics of study participants

\begin{tabular}{l|l}
\hline Kidney Blood Press Res 2019;44:984-992 \\
\hline DOI: 10.1159/000501854 & $\begin{array}{l}\text { @ 2019 The Author(s). Published by S. Karger AG, Basel } \\
\text { www.karger.com/kbr }\end{array}$ \\
\hline
\end{tabular}

Mahling et al.: SGLT2 Inhibition after Kidney Transplantation

$\begin{array}{ll}\text { Total, } n & 10 \\ \text { Age, years } & 66(56-73) \\ \text { Gender, female/male, } \% & 20 / 80 \\ \text { Living kidney donation, \% } & 80 \\ \text { Time since transplantation, years } & 5.9(4.4-8.8) \\ \text { Time since diabetes was diagnosed, years } & 18(6-33) \\ \text { Posttransplant diabetes mellitus, } \% & 40 \\ \text { Underlying chronic kidney disease, } \% & \\ \quad \text { Diabetic/hypertensive nephropathy } & 30 \\ \quad \text { IgA nephropathy } & 20 \\ \quad \text { Unspecified glomerulonephritis } & 20 \\ \quad \text { Polycystic kidney disease } & 10 \\ \quad \text { Other } & 20\end{array}$

empagliflozin for better glucose control. Our study was conducted in accordance with the Declaration of Helsinki and after approval of the Institutional Review Board of the University of Tübingen (reference 353/2016B02). All patients gave written informed consent.

\section{Study Participants}

Patients after kidney transplantation with diabetes mellitus and an estimated GFR (eGFR, as calculated by the Modification of Diet in Renal Disease - formula) of at least $45 \mathrm{~mL} / \mathrm{min} / 1.73$ $\mathrm{m}^{2}$ were considered eligible for SGLT2 inhibition. Empagliflozin treatment was started prior to inclusion in the study. Patients with type 1 diabetes or with a history of recurrent urinary tract infections were excluded from SGLT2 inhibition.

In order to provide maximal safety under SGLT2 inhibitor therapy, we reduced insulin dose by approximately $25 \%$ at first to avoid hypoglycemia. In the due course, the dose of insulin was titrated according to glucose control. Furthermore, if applicable, diuretic therapy as well as antihypertensive therapy was reduced or paused by the treating physician. Patients were informed about usual precautions including adequate oral hydration and urogenital hygiene, as well as typical signs of side effects such as urogenital infections and ketoacidosis. Regular self-blood glucose monitoring was emphasized, and 24/7 emergency contact information was given to our patients.

As part of the regular transplant center visits every 3 months, clinical and laboratory data were collected.

\section{Statistical Analysis}

All results are presented descriptively. Data are shown as median with $25-75 \%$ quartiles $\left(\mathrm{Q}_{25}-\mathrm{Q}_{75}\right)$. We performed the analysis in an intention-to-treat manner.

\section{Results}

\section{Patient Characteristics}

A total of ten kidney transplant recipients were included in our investigation (Table 1). In total, $80 \%$ received living kidney donation, $20 \%$ were transplanted via regular waiting list. Median time between transplantation and start of empagliflozin therapy was 5.9 (4.4-8.8) years. Maintenance immunosuppression consisted mainly of calcineurin inhibitors (90\%) and mycophenolate mofetil (90\%), and corticosteroids were given in 
Fig. 1. eGFR during the observation period, displayed as Boxplots with whiskers showing minimum to maximum values. eGFR, estimated glomerular filtration rate.
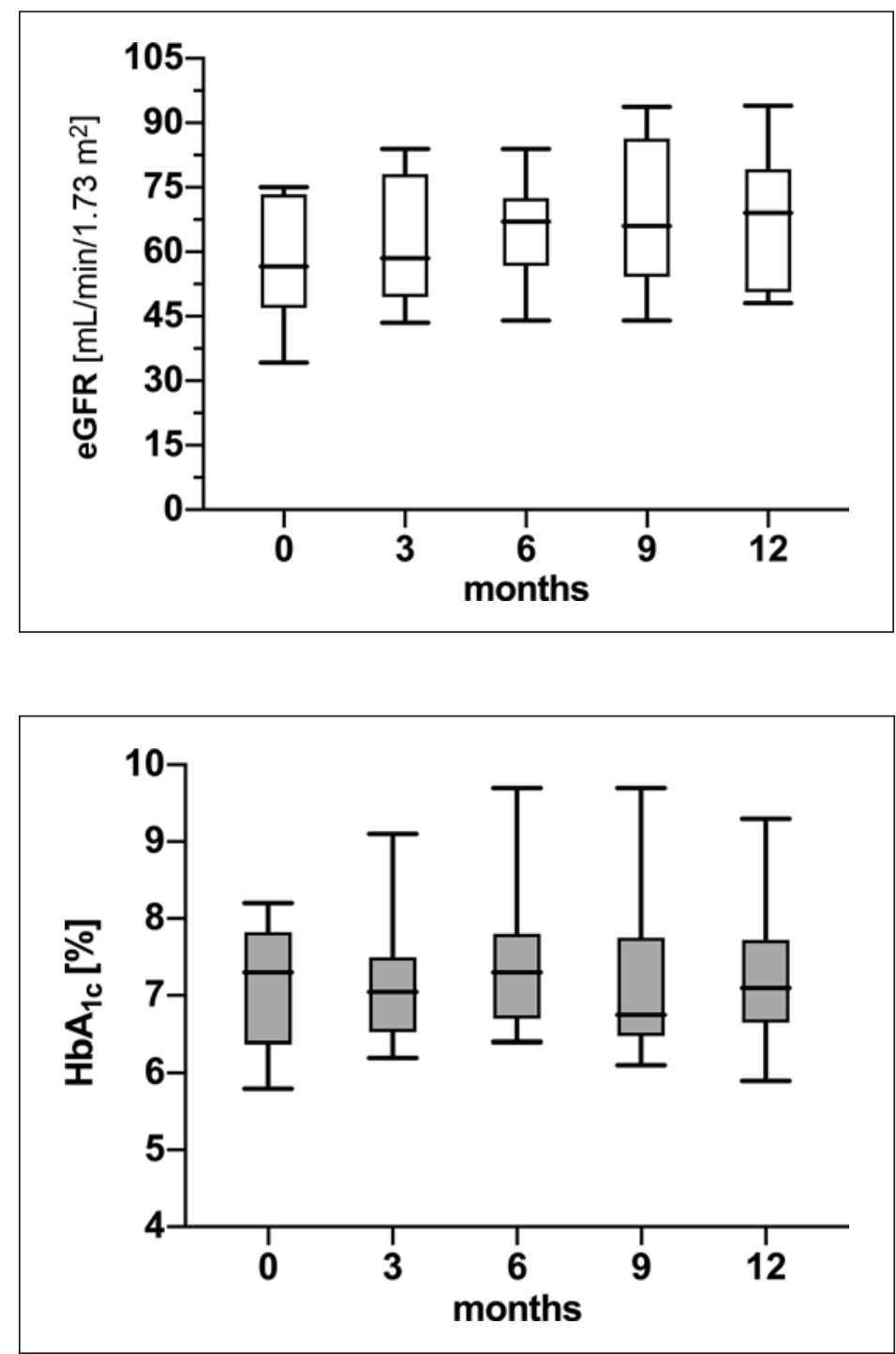

Fig. 2. $\mathrm{HbA}_{1 \mathrm{c}}$ throughout the observation period, displayed as Boxplots with whiskers showing minimum to maximum values.
$20 \%$ of patients. Prior antidiabetic therapy consisted mostly of insulin (50\%), with some patients taking metformin (20\%) or dipeptidyl peptidase-4 inhibitors (20\%). Median follow-up was 12 (5.3-12.0) months. Total observation time was 75 months, reflecting 6.3 patient-years.

\section{Kidney Allograft Function}

Baseline median eGFR was 57 (47-73) mL/min/1.73 $\mathrm{m}^{2}$. During follow-up, kidney allograft function remained stable in all of the patients. The course of the median eGFR is displayed in Figure 1.

\section{Glycemic Control}

Median $\mathrm{HbA}_{1 \mathrm{c}}$ was $7.3(6.4-7.8) \%$ at baseline compared to $7.1(6.6-7.5) \%$ at end of follow-up. The time course of the median $\mathrm{HbA}_{1 \mathrm{c}}$ is shown in Figure 2. In patients on insulin treatment, total daily insulin requirement was 10-25\% lower under SGLT2 inhibition, compared to baseline. 
Table 2. Change of selected outcomes during the observation period

\begin{tabular}{llll}
\hline Outcome & $\begin{array}{l}\text { Median value } \\
\text { at start of } \\
\text { observation }\end{array}$ & $\begin{array}{l}\text { Individual } \\
\text { absolute change, \% }\end{array}$ & $\begin{array}{l}\text { Individual } \\
\text { relative change, \% }\end{array}$ \\
\hline Body weight, kg & 75 & $-1.9(-1.9$ to 0,1$)$ & $-0.9(-2.8$ to 0.2$)$ \\
Waist circumference, cm & 110 & $-2.0(-6.0$ to 0.0$)$ & $-1.8(-6.2$ to 0.0$)$ \\
Blood pressure (systolic), mm Hg & 135 & $-2.5(-36.3$ to 0.8$)$ & $-1.4(-19.8$ to 0.6$)$ \\
Blood pressure (diastolic), mm Hg & 80 & $-0.5(-9.5$ to 7.5$)$ & $-0.6(-11.1$ to 9.4$)$ \\
Hematocrit & 38 & $+2.5(0.8$ to 4.1$)$ & $+6.5(2.3$ to 9.3$)$ \\
Uric acid, mg/dL & 5.2 & $-0.2(-0.8$ to 0.5$)$ & $-3.4(-16.5$ to 7.4$)$ \\
\hline
\end{tabular}

\section{Other Outcomes}

We found a weak reduction in the systolic blood pressure with a median of -3 ( -36 to 1 ) $\mathrm{mm} \mathrm{Hg}$. Furthermore, the body weight decreased by a median of $-1.0(-1.9$ to -0.2$) \mathrm{kg}$. Further outcomes are reported in Table 2.

\section{Adverse Events}

Urinary Tract Infections

Two patients (20\%) suffered from a urinary tract infection, corresponding to an annual incidence of 0.32 /patient/year treated with empagliflozin. During the infections, no decline in renal function was observed. Furthermore, no urosepsis was reported.

\section{Adverse Events regarding Kidney Allograft Function}

One patient (10\%) displayed acute kidney injury (AKI) stage I (rise in plasma creatinine of $0.3 \mathrm{mg} / \mathrm{dL}$ ). The calculated incidence is $0.16 /$ patient/year. Another patient displayed an episode of increased creatinine not meeting formal AKI criteria. Both episodes were rated as unlikely related to SGLT2 inhibition, and kidney function returned to baseline in both patients.

Ketoacidosis and Other Medical Events

None of our patients developed ketoacidosis during follow-up. One patient developed a small diabetic ulcer at the lower limb, which was successfully treated with local therapy while empagliflozin was continued.

Medication Discontinuation Rates

Two patients discontinued empagliflozin during the observation period on their own request. One patient felt more tired than before empagliflozin was started. The other patient suffered from a respiratory tract infection and a temporary decline in kidney allograft function (see above). Renal function returned to baseline; however, the patient wished to discontinue empagliflozin.

\section{Discussion/Conclusion}

We report prospective data on the use of SGLT2 inhibitors in kidney allograft recipients with diabetes. We demonstrate that the treatment is feasible and without significant side effects in these patients. Our data therefore provide a basis for a broader use of SGLT2 inhibitors in posttransplant populations. 


\section{Kidney \\ Blood Pressure \\ Research}

\begin{tabular}{l|l}
\hline Kidney Blood Press Res 2019;44:984-992 \\
\hline DOI: 10.1159/000501854 & $\begin{array}{l}\text { @ 2019 The Author(s). Published by S. Karger AG, Basel } \\
\text { www.karger.com/kbr }\end{array}$ \\
\hline
\end{tabular}

Mahling et al.: SGLT2 Inhibition after Kidney Transplantation

Long-term success after kidney transplantation is hindered by either progressive loss of renal function or death with a functioning graft [20], mainly due to cardiovascular complications. SGLT2 inhibitors have the potential to improve both aspects. This has impressively been shown in diabetic patients without kidney transplantation [10], and SGLT2 inhibitors have since emerged as novel nephro- and cardioprotective agents [21]. A specific benefit with the potential to modulate barriers of long-term success can therefore be expected with the use of SGLT2 inhibitors in kidney transplant recipients; however, these drugs are mostly withheld in a posttransplant setting owing to uncertainty and wariness in this special patient population. Principal concerns comprise volume homeostasis and the risk of AKI, as well as infectious complications. With our data, we now clearly demonstrate that the use is feasible and well tolerated, paving the way for novel therapeutic approaches to improve long-term outcome after kidney transplantation.

During our observation, all patients showed a stable kidney allograft function. The rate of AKI was as low as 0.16 /patient/year and limited to AKI stage I. This rate has also to be viewed in context of the easily fluctuating kidney function of kidney allograft recipients, depending on fluid homeostasis, calcineurin inhibitor trough levels, physical activity, and other environmental factors. Published literature report an incidence of 51\% AKI during approximately 4 years of follow-up in transplanted patients not treated with SGLT2 inhibitors [22]. Although limited by the low total case number, our rate and severity of AKI therefore basically reflect the regular fluctuation of allograft function observed in kidney transplant recipients. In patients without kidney transplantation, the use of SGLT2 inhibitors has not been associated with an increased risk for AKI [23], but this finding clearly cannot be easily transferred to kidney allograft recipients. From a pathophysiological point of view, the effect of SGLT2 inhibition and thereby also potential side effects of these substances are dependent on GFR and therefore self-limiting in severity with any decline in GFR. Taken together, the broader use of SGLT2 inhibitors appears feasible also in renal transplant recipients, a finding which has simultaneously been shown by Schwaiger et al. [19] as well as by Halden et al. [24], which both also report an overall stable allograft function under empagliflozin treatment. The actual incidence of AKI under treatment with empagliflozin compared to patients without SGLT2 inhibition as well as potential long-term improvement of allograft function has to be shown in larger prospective, randomized trials.

Regarding glycemic efficacy, our cohort showed a mild decrease in the median $\mathrm{HbA}_{1 \mathrm{c}}$ of $0.2 \%$. Compared with other data indicating a decrease in $\mathrm{HbA}_{1 \mathrm{c}}$ of $0.2-0.6 \%$ [10], our minor $\mathrm{HbA}_{1 \mathrm{c}}$ improvement is probably due to multiple reasons. First, we followed a cautious approach when initiating treatment with empagliflozin, as concomitant antidiabetic therapy, especially insulin, was reduced and only carefully reintroduced after a stable phase to avoid hypoglycemia. Second, the glycosuric effect in kidney transplant recipients relies on only one kidney (compared to two in nontransplant patients), reducing the amount of filtrated glucose. In a simultaneously performed randomized trial, Halden et al. [24] confirm the dependency of filtrated glucose load on GFR and report a comparable reduction in $\mathrm{HbA}_{1 \mathrm{c}}$ of $0.2 \%$ in kidney transplanted patients. Intriguingly, recent data indicate that the nephro- and cardioprotective protective effects of empagliflozin are most likely independent of the glycemic control [25] but probably a consequence of multiple factors such as reduction of glomerular capillary pressure and hyperfiltration, improvement of blood pressure, and direct molecular effects, for example, reduction of cardiac inflammation and oxidative stress [26]. Another hypothesis to explain the glucose-independent protective effects of SGLT2 inhibition is the normalization of pathologic sympathetic nervous activity [25]. In this respect, kidney transplant recipients comprise an interesting patient population, as transplanted kidneys are denervated and the lack of autonomic regulation might modulate the effects of SGLT2 inhibition. Our study was not designed to investigate this effect, but further investigations in kidney transplanted 


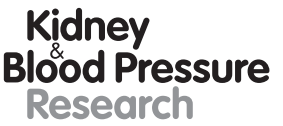

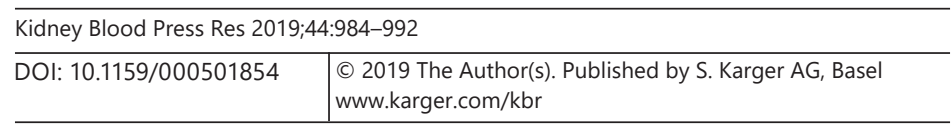

Mahling et al.: SGLT2 Inhibition after Kidney Transplantation

patients could include data on autonomic activity and thus discern a potential contribution of sympathetic activity. Taken together, the addition of SGLT2 inhibitors seems to be important irrespective of glucose control and is currently also investigated in patients without diabetes [27].

We observed urinary tract infections in two of our patients, reflecting an annual incidence of 0.32 /patient. The infection course was uncomplicated in all patients and did not result in discontinuation of empagliflozin. Again, this rate is not high for kidney transplant recipients, as Ariza-Heredia et al. [28] report a comparable incidence of 34\% during an observation period of two years. In the EMPA-REG trial, the only significant side effect was found for genital infections, which were significantly more likely in patients receiving empagliflozin $[11,17]$. We did not detect any genital infection during follow-up. Furthermore, none of our patients developed ketoacidosis, a feared side effect of SGLT2 inhibition [17].

Our data do yield limitations: As our study was observational, neither patients nor physicians were blinded to the treatment and our investigation lacks randomization as well as a comparator group. Also, our study population comprised well-selected patients from our routine outpatient care center at this time-point with high adherence to our recommendations and ability to react adequately in the case of a possible adverse effect. A clear strength, however, is the long prospective period of observation of one year.

In conclusion, we report prospective data clearly indicating that treatment with empagliflozin is feasible in selected kidney transplant recipients with diabetes with a good safety profile. Although our case series includes only a limited number of patients, we report data with a median follow-up of 12 months. These data provide a basis for prospective randomized trials to investigate the long-term benefits on allograft function and cardiovascular risk in kidney transplant recipients.

\section{Acknowledgments}

The authors thank all participating patients and relatives for their participation. Further, we thank Cristina Teixeira for her excellent support throughout the study. We acknowledge the support by Deutsche Forschungsgemeinschaft and Open Access Publishing Fund of the University of Tübingen.

\section{Statement of Ethics}

Our study was conducted in accordance with the Declaration of Helsinki and after approval of the Institutional Review Board of the University of Tübingen (reference 353/ 2016B02). All patients gave written informed consent.

\section{Disclosure Statement}

Prof. Dr. Andreas Fritsche is a member of a national scientific advisory board of Boehringer. The other authors of this manuscript have no conflicts of interest to disclose.

\section{Funding Sources}

None. 


\section{Kidney \\ Blood Pressure \\ Research}

\begin{tabular}{l|l}
\hline Kidney Blood Press Res 2019;44:984-992 \\
\hline DOI: 10.1159/000501854 & $\begin{array}{l}\text { ○ 2019 The Author(s). Published by S. Karger AG, Basel } \\
\text { www.karger.com/kbr }\end{array}$ \\
\hline
\end{tabular}

Mahling et al.: SGLT2 Inhibition after Kidney Transplantation

\section{Author Contributions}

M.M. and M.G.: conceived the work, acquired data, interpreted results, drafted manuscript, provided intellectual content, approved the final version. A.S.: acquired data, interpreted results, revised manuscript, provided intellectual content, approved the final version. S.N. and A.F.: interpreted results, revised manuscript, provided intellectual content, approved the final version. N.H.: conceived the work, acquired data, interpreted results, revised manuscript, provided intellectual content, approved the final version.

\section{References}

1 United States Renal Data System. 2017 USRDS annual data report: Epidemiology of kidney disease in the United States. National Institutes of Health, National Institute of Diabetes and Digestive and Kidney Diseases, Bethesda, MD, 2017.

2 Cooper L, Oz N, Fishman G, Shohat T, Rahamimov R, Mor E, et al. New onset diabetes after kidney transplantation is associated with increased mortality-A retrospective cohort study. Diabetes Metab Res Rev. 2017 Nov; 33(8):e2920.

3 Sharif A, Baboolal K. Complications associated with new-onset diabetes after kidney transplantation. Nat Rev Nephrol. 2011 Nov;8(1):34-42.

4 Burroughs TE, Swindle J, Takemoto S, Lentine KL, Machnicki G, Irish WD, et al. Diabetic complications associated with new-onset diabetes mellitus in renal transplant recipients. Transplantation. 2007 Apr;83(8): 1027-34.

5 Parekh J, Bostrom A, Feng S. Diabetes mellitus: a risk factor for delayed graft function after deceased donor kidney transplantation. Am J Transplant. 2010 Feb;10(2):298-303.

6 Siedlecki A, Irish W, Brennan DC. Delayed graft function in the kidney transplant. Am J Transplant. 2011 Nov; 11(11):2279-96.

7 Sharif A, Cohney S. Post-transplantation diabetes-state of the art. Lancet Diabetes Endocrinol. 2016 Apr;4(4): 337-49.

8 Guthoff M, Wagner R, Weichbrodt K, Nadalin S, Königsrainer A, Häring HU, et al. Dynamics of Glucose Metabolism After Kidney Transplantation. Kidney Blood Press Res. 2017;42(3):598-607.

9 Gallo LA, Wright EM, Vallon V. Probing SGLT2 as a therapeutic target for diabetes: basic physiology and consequences. Diab Vasc Dis Res. 2015 Mar;12(2):78-89.

10 Zinman B, Wanner C, Lachin JM, Fitchett D, Bluhmki E, Hantel S, et al.; EMPA-REG OUTCOME Investigators. Empagliflozin, Cardiovascular Outcomes, and Mortality in Type 2 Diabetes. N Engl J Med. 2015 Nov;373(22): 2117-28.

11 Wanner C, Inzucchi SE, Lachin JM, Fitchett D, von Eynatten M, Mattheus M, et al.; EMPA-REG OUTCOME Investigators. Empagliflozin and Progression of Kidney Disease in Type 2 Diabetes. N Engl J Med. 2016 Jul;375(4): 323-34.

12 Wanner C, Cooper ME, Inzucchi SE, Zinman B, Hehnke U, von Eynatten M, et al. Empagliflozin Improves Kidney Outcomes Irrespective of Control of Blood Pressure, Low-Density Lipoprotein Cholesterol and HbA1c [abstract]. German Society of Nephrology 10th Annual Congress 2018 (DGFN 2018). Berlin, 2018.

13 Skrtić M, Yang GK, Perkins BA, Soleymanlou N, Lytvyn Y, von Eynatten M, et al. Characterisation of glomerular haemodynamic responses to SGLT2 inhibition in patients with type 1 diabetes and renal hyperfiltration. Diabetologia. 2014 Dec;57(12):2599-602.

14 Cherney DZ, Perkins BA, Soleymanlou N, Maione M, Lai V, Lee A, et al. Renal hemodynamic effect of sodiumglucose cotransporter 2 inhibition in patients with type 1 diabetes mellitus. Circulation. 2014 Feb;129(5):587-97.

15 Mayer G, Wanner C, Weir M, Inzucchi SE, Koitka-Weber A, Hantel S, et al. Analysis from the EMPA-REG OUTCOME $^{\circledR}$ trial indicates empagliflozin may assist in preventing the progression of chronic kidney disease in patients with type 2 diabetes irrespective of medications that alter intrarenal hemodynamics. Kidney Int. 2019 Mar 21. pii: S0085-2538(19)30317-5.

16 Neal B, Perkovic V, Mahaffey KW, de Zeeuw D, Fulcher G, Erondu N, et al. Canagliflozin and Cardiovascular and Renal Events in Type 2 Diabetes. N Engl J Med. 2017;377:644-57.

17 Wiviott SD, Raz I, Bonaca MP, Mosenzon O, Kato ET, Cahn A, et al.; DECLARE-TIMI 58 Investigators. Dapagliflozin and Cardiovascular Outcomes in Type 2 Diabetes. N Engl J Med. 2019 Jan;380(4):347-57.

18 Halimi S, Vergès B. Adverse effects and safety of SGLT-2 inhibitors. Diabetes Metab. 2014 Dec; 40 (6 Suppl 1):S28-34.

19 Schwaiger E, Burghart L, Signorini L, Ristl R, Kopecky C, Tura A, et al. Empagliflozin in posttransplantation diabetes mellitus: A prospective, interventional pilot study on glucose metabolism, fluid volume, and patient safety. Am J Transplant. 2019 Mar;19(3):907-19.

20 Briganti EM, Russ GR, McNeil JJ, Atkins RC, Chadban SJ. Risk of renal allograft loss from recurrent glomerulonephritis. N Engl J Med. 2002 Jul;347(2):103-9. 
21 Zelniker TA, Wiviott SD, Raz I, Im K, Goodrich EL, Bonaca MP, et al. SGLT2 inhibitors for primary and secondary prevention of cardiovascular and renal outcomes in type 2 diabetes: a systematic review and meta-analysis of cardiovascular outcome trials. Lancet. 2019 Jan 5;393(10166):31-9.

22 Nagarajan M, Ramanathan S, Dhanapriya J, Dineshkumar T, Subramaniyan TB, Gopalakrishnan N. Impact of acute kidney injury on renal allograft survival. Ren Fail. 2017 Nov;39(1):40-4.

23 Nadkarni GN, Ferrandino R, Chang A, Surapaneni A, Chauhan K, Poojary P, et al. Acute Kidney Injury in Patients on SGLT2 Inhibitors: A Propensity-Matched Analysis. Diabetes Care. 2017 Nov;40(11):1479-85.

24 Halden TA, Kvitne KE, Midtvedt K, Rajakumar L, Robertsen I, Brox J, et al. Efficacy and Safety of Empagliflozin in Renal Transplant Recipients With Posttransplant Diabetes Mellitus. Diabetes Care. 2019 Jun;42(6):106774.

25 Inzucchi SE, Kosiborod M, Fitchett D, Wanner C, Hehnke U, Kaspers S, et al. Improvement in Cardiovascular Outcomes With Empagliflozin Is Independent of Glycemic Control. Circulation. 2018 Oct;138(17):1904-7.

26 Lahnwong S, Chattipakorn SC, Chattipakorn N. Potential mechanisms responsible for cardioprotective effects of sodium-glucose co-transporter 2 inhibitors. Cardiovasc Diabetol. 2018 Jul;17(1):101.

27 Effects of Dapagliflozin in Non-diabetic Patients With Proteinuria. ClinicalTrials.gov [Internet]. [cited 2019 Jan 5]. Available from: https://clinicaltrials.gov/ct2/show/NCT03190694.

28 Ariza-Heredia EJ, Beam EN, Lesnick TG, Kremers WK, Cosio FG, Razonable RR. Urinary tract infections in kidney transplant recipients: role of gender, urologic abnormalities, and antimicrobial prophylaxis. Ann Transplant. 2013 May; 18:195-204. 